\title{
ON UPPER GAUGE DENSITY
}

\author{
H. W. PU AND H. H. PU
}

\begin{abstract}
Let $g$ be a diametric gauge over a metric space $(X, \rho)$. It is proved, in this paper, that the upper gauge density $D(A, x)=0$ for almost all points of the complement of $A$ provided that $A$ is in a certain family which contains all Borel sets of finite measure. Also, a relation between conditions for a diametric gauge and certain regularity conditions is given.
\end{abstract}

1. Introduction. Let $(X, \rho)$ be a metric space, $\varsigma$ be a family of subsets of $X$ with the empty set $\varnothing$ in it, and let $g$ be a gauge function over $X$ with domain $\delta$, that is, $g$ is nonnegative, real-valued and $g(\varnothing)=0$. The outer measure $m$ defined by

$$
m(A)=\lim _{r \rightarrow 0+} \inf \left\{\sum_{i=1}^{\infty} g\left(S_{i}\right) \mid A \subset \bigcup_{i=1}^{\infty} S_{i}, d\left(S_{i}\right)<r, S_{i} \in \mathcal{\delta}\right\}
$$

for all $A \subset X$, where $d\left(S_{i}\right)$ denotes the diameter of the set $S_{i}$, is called the outer measure generated by the gauge $g$. We shall denote the complement of a set $A \subset X$ by $\tilde{A}$, and we say that $A$ is measurable if, for all $B \subset X$,

$$
m(B)=m(B \cap A)+m(B \cap \tilde{A}) .
$$

The upper gauge density of a set $A$ at a point $x$ is defined to be

$$
D(A, x)=\lim _{r \rightarrow 0+} \sup \left\{\frac{m(A \cap S)}{g(S)} \mid x \in S \in \Omega, d(S)<r, g(S) \neq 0\right\} .
$$

Eames assumed that $\left(\mathrm{e}_{1}\right)$ every set $S$ in the family $\varsigma$ is closed in $X,\left(\mathrm{e}_{2}\right)$ $g(S)=0$ implies $S=\varnothing$ and $\left(\mathrm{e}_{3}\right)$ for every set $A \subset X$ and every $r>0$, there exists a sequence $\left\{S_{i}\right\}$ in $S$ such that $A \subset \cup S_{i}$ and $d\left(S_{i}\right)<r$. He proved that if $m(A)<\infty$, then $D(A, x) \geqslant 1$ for almost all $x \in A$ [1, Theorem 6]. Under additional conditions which he called the regularity conditions, he proved that if $m(A)<\infty$, then $D(A, x)=1$ for almost all $x \in A[1$, Theorem 9] and that if $m(A)<\infty$ and $B$ is separated from $A$, then $D(A, x)=0$ for almost all $x \in B$ [1, Theorem 8]. This together with the fact that if $A$ is measurable and $m(A)<\infty$, then $\tilde{A}$ is separated from $A$ [1, Theorem 3] gives the result that if $A$ is measurable and $m(A)<\infty$, then $D(A, x)=0$ for almost all $x \in \tilde{A}$. Later, Freilich proved Eames' Theorem 6 under more general conditions [2, Theorems 3.2, 3.3] and by imposing some

Received by the editors March 27, 1975.

AMS (MOS) subject classifications (1970). Primary 28A10, 28 A15.

Key words and phrases. Outer measure, upper gauge density.

(1) American Mathematical Society 1976 
other conditions under which he called $g$ a diametric gauge, he obtained Eames' Theorem 9 [2, Theorem 4.4], but $D(A, x)$ for $x \in \tilde{A}$ was not investigated in his paper. It is natural to ask if Eames' result for $D(A, x)$ with $x \in \tilde{A}$ holds under Freilich's conditions.

2. Diametric gauge and the regularity conditions. The regularity conditions that Eames used for his Theorems 8 and 9 read as follows : For every $S \in \Sigma$, there exists $S^{\prime} \in \mathcal{S}$ such that

(i) $S^{\prime} \supset\{x \mid \rho(x, S) \leqslant \alpha d(S)\}$, where $\alpha$ is a finite number greater than 1 and independent of $S$,

(ii) $g\left(S^{\prime}\right) \leqslant \beta g(S)$, where $\beta$ is a finite number independent of $S$,

(iii) for every $r>0$ there is an $\eta>0$ such that $d(S)<\eta$ implies $d\left(S^{\prime}\right)$ $<r$.

It should be noted that Eames' proof works equally well if these conditions hold only for $S \in \mathcal{S}$ with $d(S)$ sufficiently small. We shall mean this weakened form whenever we say the regularity conditions.

The gauge $g$ is called diametric if

(1) there exists a $\delta>0$ such that if $x \in X, 0<r \leqslant \delta$, then $U(x, r) \in \S$ and $d(U(x, r))>r$, where $U(x, r)=\{y \in X \mid \rho(x, y)<r\}$,

(2) every set $S$ in $S$ is measurable,

$\left(3^{\prime}\right)$ there exist nondecreasing functions $f_{1}$ and $f_{2}$ on the nonnegative real numbers and a positive constant $\lambda$ such that $f_{1}(2 r) \leqslant \lambda f_{2}(r)$ and if $S \in \Omega$, $d(S)=r$, then $f_{2}(r) \leqslant g(S) \leqslant f_{1}(r)$,

(4) there exists a positive integer $k$ such that any open sphere of radius $6 r$ can be covered by $k$ open spheres of radius $r$ ( $k$ independent of $r$ ).

Freilich made a remark that in the above condition ( $\left.3^{\prime}\right) 2 r$ can be replaced by $p r$ with any fixed $p>1$. In the following, we shall use, instead of $\left(3^{\prime}\right)$ above, the condition:

(3) there exist nondecreasing functions $f_{1}$ and $f_{2}$ on the nonnegative real numbers and a positive constant $\lambda$ such that $f_{1}(p r) \leqslant \lambda f_{2}(r)$ and if $S \in S$, $d(S)=r$, then $f_{2}(r) \leqslant g(S) \leqslant f_{1}(r)$, where $p$ is a fixed number greater than 4.

With this permissible change, we find a relation between a diametric gauge and the regularity conditions.

THEOREM 2.1. If $g$ is a diametric gauge, then the regularity conditions are satisfied.

Proof. Suppose that conditions (1), (2) and (3) are satisfied (note that we do not need condition (4) at all). Since $p / 4>1$, we may choose $\alpha=p / 4$ and $\beta=\lambda$. For any given $S \in \mathcal{S}$ with $d(S)<2 \delta / p$, it can be easily checked that $S^{\prime}=U\left(x_{0}, p d(S) / 2\right)$ is a set in $S$ such that conditions (i), (ii) and (iii) are fulfilled, where $x_{0}$ is any point in $S$ (the case $S=\varnothing$ is trivial).

REMARK 2.2. In view of condition (3) and the fact $g(\varnothing)=0$, we see that $f_{1}(0)=f_{2}(0)=0$. Also, if $f_{1}(r)$ or $f_{2}(r)$ vanishes at some $r>0$, then $m(A)=0$ or $\infty$ for every $A \subset X$. This case is of no interest. Thus we consider only the case that $f_{1}(r)$ and $f_{2}(r)$ are positive if $r>0$. It follows that $g(S)=0$ if and only if $d(S)=0$.

REMARK 2.3. Eames' sequential covering assumption $\left(\mathrm{e}_{3}\right)$ can be eliminated 
by the convention that $m(A)=\infty$ if there exists no sequence $\left\{S_{i}\right\}$ in $\delta$ such that $A \subset \cup S_{i}$ and $d\left(S_{i}\right)<r$ for some $r>0$. The assumption $\left(\mathrm{e}_{2}\right)$ is used in the proof of his Theorems 8 and 9 but $\left(\mathrm{e}_{1}\right)$ is not used for these theorems. Owing to Remark 2.2 above, a simple modification of his proof can go through under Freilich's conditions (1), (2) and (3). Therefore, Eames' method can be used to prove Freilich's Theorem 4.4 which has the same conclusion as Eames' Theorem 9. It is worth noting that Freilich's proof is of great length while Eames' proof, by using the axiom of choice or Zorn's lemma, is short. Besides this, condition (4) is not needed. Is there a proof for the same result under conditions (1), (2) and (3) without using the axiom of choice?

REMARK 2.4. The closedness assumption $\left(e_{1}\right)$ is used in Eames' paper, as far as we are concerned, only in the proof of his Theorem 3 and it is easily seen from his proof that this condition can be replaced by: every set $S$ in $\mathcal{S}$ is an $F_{\sigma}$-set in $X$. Therefore, as we mentioned in the introduction about combining Eames' Theorems 3 and 8, we obtain the following result which we shall use in the next section.

Let $g$ be a gauge over $(X, \rho)$ with domain $\delta$ in which every set is an $F_{\sigma}$-set in $X$ such that conditions (1), (2) and (3) are satisfied. If $A \subset X$ and $m(A)<\infty$, then measurability of $A$ implies that $D(A, x)=0$ for almost all $x \in \tilde{A}$. Furthermore, the converse implication is then guaranteed by Eames' Theorem 11.

3. Main result. To keep the conditions general (by this we mean conditions (1), (2), (3) and no more), we shall not assume that every set in $\delta$ is a certain sort but we make use of a subfamily $\mathcal{Q}$ of $\delta$ and introduce a new outer measure $\mu$. In order to avoid confusion, we use the notations $D_{m}(A, x)$ and $D_{\mu}(A, x)$ to mean the different upper gauge densities. More precisely, let

$$
\text { थ }=\{U(x, r) \mid x \in X, 0<r \leqslant \delta\} \cup\{\varnothing\},
$$

where $\delta$ is the number in condition (1), and let $\mu$ be the outer measure generated by the gauge function $g \mid \mathcal{Q} l$, that is, for $A \subset X$,

$$
\mu(A)=\lim _{r \rightarrow 0+} \inf \left\{\sum_{i=1}^{\infty} g\left(U_{i}\right) \mid A \subset \bigcup_{i=1}^{\infty} U_{i}, d\left(U_{i}\right)<r, U_{i} \in \mathcal{Q}\right\} .
$$

Also, $D_{m}(A, x)$ is the number given by (1.2) while

$$
\begin{aligned}
D_{\mu} & (A, x) \\
\quad & =\lim _{r \rightarrow 0+} \sup \left\{\frac{\mu(A \cap U)}{g(U)} \mid x \in U \in \mathcal{Q}, d(U)<r, g(U) \neq 0\right\} .
\end{aligned}
$$

In the following lemmas, $\lambda$ is the positive number in condition (3).

$$
\text { Lemma 3.1. For every } A \subset X, m(A) \leqslant \mu(A) \leqslant \lambda m(A) \text {. }
$$

Proof. The inequality $m(A) \leqslant \mu(A)$ is obvious from (1.1) and (3.1). For the other inequality, we may assume that $m(A)<\infty$. Thus there exists, for each sufficiently small $r>0$, a sequence $\left\{S_{i}\right\}$ in $\delta$ covering $A$ with $d\left(S_{i}\right)$ $<r$. From the proof for Theorem 2.1 , we see easily that $\mu(A) \leqslant \lambda m(A)$. 
Corollary. $m(A)<\infty$ if and only if $\mu(A)<\infty . m(A)=0$ if and only if $\mu(A)=0$. Hence the terms "of finite measure", "almost all" and "null" can be safely used without confusion.

LeMMA 3.2. For every $A \subset X$ and every $x \in X, \lambda^{-1} D_{m}(A, x) \leqslant$ $D_{\mu}(A, x) \leqslant \lambda D_{m}(A, x)$.

Proof. The inequality $D_{\mu}(A, x) \leqslant \lambda D_{m}(A, x)$ is obvious from (1.2) and (3.2), and the inequality $\lambda^{-1} D_{m}(A, x) \leqslant D_{\mu}(A, x)$ can be proved by viewing the proof for Theorem 2.1 and using the first inequality in Lemma 3.1 above.

Corollary. $D_{m}(A, x)=0$ if and only if $D_{\mu}(A, x)=0$.

It is clear that $\mathscr{U}$ and $g \mid \mathcal{U}$ satisfy conditions (1) and (3). In fact, condition (2) is also satisfied (here measurability is, of course, with respect to $\mu$ ) $[3, \mathrm{p}$. 52]. Moreover, every set in $\mathscr{Q}$ is an $F_{\sigma}$-set. By Remark 2.4 , for $A \subset X$ with $\mu(A)<\infty$, we have the following result.

LEMMA 3.3. $A$ is $\mu$-measurable if and only if $D_{\mu}(A, x)=0$ for almost all $x \in \tilde{A}$.

From this lemma and the above corollaries, we obtain the result mentioned in the abstract.

THEOREM 3.4. If $A \subset X$ is $\mu$-measurable (in particular, if $A$ is Borel) and $m(A)<\infty$, then $D_{m}(A, x)=0$ for almost all $x \in \tilde{A}$.

Finally, for $A \subset X$ with $m(A)<\infty$, let $G=\{x \mid x \in \tilde{A}, D(A, x)>0\}$. We state a theorem similar to Eames' Theorem 11. The proof is also similar to his and is omitted here.

THEOREM 3.5. (a) $A \cup G$ is m-measurable,

(b) if $G$ is null, then $A$ is m-measurable.

Since Eames' result shows that $G$ is null if and only if $A$ is $\mu$-measurable, we get the following

Corollary. Every $\mu$-measurable set with finite measure is also $\mathrm{m}$ measurable.

\section{REFERENCES}

1. W. Eames, A local property of measurable sets, Canad. J. Math. 12(1960), 632-640. MR 23 \# A285.

2. G. Freilich, Gauges and their densities, Trans. Amer. Math. Soc. 122(1966), 153-162. MR 34 \#6018.

3. S. Saks, Theory of the integral, Monografie Mat., vol. VIi, PWN, Warsaw, 1937; reprint, Dover, New York, 1964. MR 29 \#4850.

Department of Mathematics, Texas A\&M University, College Station, Texas 77843

Department of Mathematics, Soochow University, Taipei, Republic of China 